\title{
The first study of 54 new eccentric eclipsing binaries in our Galaxy ${ }^{\star}$
}

\author{
P. Zasche ${ }^{1}$, M. Wolf ${ }^{1}$, R. Uhlař ${ }^{2}$ P. Cagaš ${ }^{3}$, J. Juryšek ${ }^{4}$, M. Mašek ${ }^{4,5}$, K. Hoňková ${ }^{5}$, H. Kučáková ${ }^{1}$, M. Lehký ${ }^{6,7}$, \\ L. Kotková ${ }^{8}$, G. J. White ${ }^{9,10}$, D. Bewsher ${ }^{11}$, M. Tylšar ${ }^{12}$, M. Jelínek ${ }^{8,13}$, and A. Paschke ${ }^{14}$
}

\author{
1 Astronomical Institute, Charles University, Faculty of Mathematics and Physics, 18000 Praha 8, V Holešovičkách 2, \\ Czech Republic \\ e-mail: zasche@sirrah.troja.mff.cuni.cz \\ 2 Private Observatory, Pohoří 71, 25401 Jílové u Prahy, Czech Republic \\ BSObservatory, Modrá 587, 76001 Zlín, Czech Republic \\ Institute of Physics, Czech Academy of Sciences, Na Slovance 1999/2, 18221 Praha 8, Czech Republic \\ 5 Variable Star and Exoplanet Section of the Czech Astronomical Society, Vsetínská 941/78, 75701 Valašské Meziříčí, \\ Czech Republic \\ 6 Úpice Observatory, U Lipek 160, 54232 Úpice, Czech Republic \\ 7 Astronomical Society Hradec Králové, Zámeček 456/30, 50008 Hradec Králové, Czech Republic \\ 8 Astronomical Institute, The Czech Academy of Sciences, Fričova 298, 25165 Ondřejov, Czech Republic \\ 9 School of Physical Sciences, The Open University, Walton Hall, Milton Keynes MK6 7AA, UK \\ 10 RAL Space, The Rutherford Appleton Laboratory, Chilton, Didcot, Oxfordshire OX11 0NL, UK \\ 11 Jeremiah Horrocks Institute, University of Central Lancashire, Preston PR1 2HE, UK \\ 12 Hvězdárna, Kolářovy sady 3348, 79601 Prostějov, Czech Republic \\ 13 Instituto de Astrofísica de Andalucía, PO Box 03004, 18080 Granada, Spain \\ 14 Weierstr 30, 8630 Rueti, Switzerland
}

Received 8 February 2018 / Accepted 28 May 2018

\begin{abstract}
We present an analysis of the apsidal motion and light curve parameters of 54 never-before-studied galactic Algol-type binaries. This is the first analysis of such a large sample of eccentric eclipsing binaries in our Galaxy, and has enabled us to identify several systems that are worthy of further study. Bringing together data from various databases and surveys, supplemented with new observations, we have been able to trace the long-term evolution of the eccentric orbit over durations extending back up to several decades. Our present study explores a rather different sample of stars to those presented in the previously published catalogue of eccentric eclipsing binaries, sampling to fainter magnitudes, covering later spectral types, sensitive to different orbital periods with more than $50 \%$ of our systems having periods longer than six days. The typical apsidal motion in the sample is rather slow (mostly of order of centuries long), although in some cases this is less than $50 \mathrm{yr}$. All of the systems, except one, have eccentricities less than 0.5 , with an average value of 0.23 . Several of the stars also show evidence for additional period variability. In particular we can identify three systems in the sample, HD 44093, V611 Pup, and HD 313631, which likely represent relativistic apsidal rotators.
\end{abstract}

Key words. binaries: eclipsing - stars: fundamental parameters - stars: general

\section{Introduction}

For independent testing of general relativity, as well in confronting stellar structure models, eccentric eclipsing binary systems are often mentioned as being ideal astrophysical laboratories. However, in the last few decades the exploitation of eccentric eclipsing binaries (EEBs) to current astrophysical research problems has been developed, thanks to the large photometric surveys and availability of precise data, including for fainter targets. Studies have focused on, in particular, the periodeccentricity distribution of the binary systems, which provides a crucial test of our models of star formation, theory of orbital circularization, and dynamical evolution of binaries and multiple systems, amongst others. These studies have asked questions such as: Is the formation of binary and multiple star systems

\footnotetext{
* The full Table 1 is only available at the CDS via anonymous ftp to cdsarc.u-strasbg. fr (130.79.128.5) or via http://cdsarc.
} u-strasbg.fr/viz-bin/qcat?]/A+A/619/A85 solely a matter of fragmentation, accretion, or some N-body dynamics, or is it a fruitful combination of all these mechanisms? Is the subsequent orbit migration crucial for explaining the observed orbital properties of binaries and multiples? The distribution of eccentricity can provide strong constraints on star forming theories and can be compared with stellar formation models and N-body simulations (see e.g. Tokovinin 2008, or Kiminki \& Kobulnicky 2012). The number of known systems suitable for detailed modelling is still rather limited, and studies like the present one significantly extend the available sample and help us to answer some of the questions above.

Study of the light curves (LCs) of eclipsing binaries and their modelling with available tools (i.e. a well-known WilsonDevinney algorithm, see Wilson \& Devinney 1971 and Wilson 1979) can be used for deriving the value of orbital eccentricity. On the other hand, the orbital period is mostly a known quantity. Moreover, the LC modelling also provides information about the physical properties of both components, their relative 
luminosities or relative radii (with respect to the semimajor axis). Additionally, the results of such an analysis can help to estimate the internal structure constants for the particular system.

The investigation of period changes in EEB systems on the basis of their minima timings variation (both primary and secondary) alone is a familiar method in stellar astrophysics as described in several seminal papers, for example, Giménez \& García-Pelayo (1983), or Giménez \& Bastero (1995). As a very short description of the method, the sidereal and anomalistic periods of the binary are connected with the relation $P_{\mathrm{s}}=P_{\mathrm{a}}(1-\dot{\omega} / 2 \pi)$, where $\dot{\omega}$ is the rate of the apsidal motion $\omega=\omega_{0}+\dot{\omega} E$. The period of such revolution is then $U=2 \pi P_{\mathrm{a}} / \dot{\omega}$. The individual equations for the computation of the time of primary and secondary minima were published, for example in Giménez \& García-Pelayo (1983). We also suppose that the apsidal motion remains linear in time.

The number of new photometric observations of eclipsing binaries is increasing rapidly year-on-year. This is partly due to non-professional astronomers, but mostly because of new photometric surveys covering the whole sky. Using these data, it is possible to derive many new times of eclipses at various time epochs, and to trace periodic changes in these systems, that is, detect the third bodies, or study the apsidal motion in them. Examples of such studies have been published quite frequently during the last few years, see for example Pilecki et al. (2007), Zasche et al. (2014a), or Borkovits et al. (2016).

However, despite increasing the number of data points, a detailed analysis is still lacking for some of the systems. Hence, for our analysis we have used observations from automated photometric surveys, from satellites, from our new ground-based observations, as well as from previously published data from various publications.

The advantage of our method for deriving the eccentricity is obvious. Without having any information about the masses (no spectroscopy and RV solutions available for these systems), our solution still has to be considered preliminary. However, having an estimate of the eccentricity from the LC solution, it becomes possible to perform a period analysis of the eclipse times to refine its value, and vice versa. Sometimes the LC coverage is insufficient to derive a reliable value of the eccentricity, but the $\mathrm{O}-\mathrm{C}$ diagram with times of eclipses can help us. Sometimes the change of $\omega$ is so slow that a trustworthy analysis cannot be done using only minima times, but the LC solution can reveal the eccentricity more reliably.

For our target selection the selected systems should not have been studied before, meaning that no LC or period analysis has been published. Although some of the systems have had their orbital periods mentioned in previous publications, no further information or analysis was available. All the systems are well detached (as needed for an eccentric-orbit binary), located in both the southern and northern hemispheres, and their respective orbital periods range from several days to several dozen days. The only input parameters needed for the analysis are the period and an estimate of the primary temperature (see below). In the whole study, we see rather heterogeneous effort from the various observers (mainly professionals, but sometimes also nonprofessionals) and observational strategies (sometimes dedicated observations, sometimes only by chance products of observing another target in the field). Some of the selected stars are relatively faint, although several of these systems are sufficiently bright $(\mathrm{mag}<10)$ that they were observed by the HIPPARcos satellite (ESA 1997). We consider the present paper as a starting point for some future, more focused, observational effort, or to aid the target selection for a future dedicated studies of these targets.
An overview of the basic parameters of the analysed systems is presented in Table A.1, where the individual values are taken from the available databases and catalogues (SIMBAD, GCVS - Samus et al. 2017). The primary temperature needed for the LC analysis was in most cases estimated from the photometric indices, but for those systems for which a spectral classification has been published, this was used for a temperature estimation of the fixed $T_{1}$ value used for the LC analysis. For most systems, the GCVS designation is still missing, hence we used some most common catalogue information taken from CDS/SIMBAD, together with the precise J2000.0 coordinates for better identification of the star.

For analysis of the LC as well as deriving new minima times, several different sources and databases were used together. These data, along with our new observations, present material that is sometimes very suitably complementary. For example, sometimes the phase coverage is poor in one source, but it may have good photometry in another, and vice versa. See our final plots below in Fig. A.1.

\section{Approach for the analysis}

Despite the fact that several of the systems have rather low photometric variation amplitudes, and some are also relatively faint targets, usually some of the automatic photometric surveys can provide us with reliable photometry to facilitate LC modelling. Hence, when having different sources of data from different surveys, databases, and publications, we used the best of these for the LC solution (i.e. those with the lowest scatter and the best phase coverage of the complete light curve).

For the light-curve analysis we used the code PHOEBE (Prša \& Zwitter 2005), which is originally based on the WilsonDevinney algorithm (Wilson \& Devinney 1971; Wilson 1979) and its later modifications. For those systems where photometry in different filters was used, we analysed these data simultaneously.

Because spectroscopic studies with radial velocities are missing for all these systems, there are several assumptions which have to be considered. At first, the mass ratio of all the systems was kept at a value of unity. This approach is justified because all the systems are well-detached and the ellipsoidal variations outside of their minima are almost negligible. For such systems the photometric mass ratio can only be approximately estimated, as quoted for example by Terrell \& Wilson (2005). Other physical parameters were instead estimated and derived in a relative sense, rather than in absolute units (e.g. radii, luminosities), hence these values are still only approximate estimates and should not be used as fundamental parameter sources.

Due to all these reasons we used the following approach for the analysis. Firstly, a very first rough LC analysis was performed. Second, the initial LC analysis was used to estimate the available minima, which were then analysed to estimate preliminary apsidal motion parameters (with the assumption $i=90^{\circ}$ ). Third, the eccentricity $(e)$, argument of periastron $(\omega)$ and apsidal motion rate $(\dot{\omega})$ resulting from the apsidal motion analysis were used for the preliminary light curve analysis. Fourth, a further parameter from the LC analysis, the inclination ( $i)$, was then used for the apsidal motion analysis. And finally, the resulting $e$, $\omega$, and $\dot{\omega}$ values from the apsidal motion analysis were used for the final LC analysis.

The automatic fitting procedure (AFP) method for deriving the individual times of minima was the same as presented in Zasche et al. (2014b), applied to different photometric databases. All the minima times used for the analysis were given in Table 1. 
Table 1. Heliocentric minima of the systems used for our analysis.

\begin{tabular}{lllcll}
\hline \hline Star & HJD - 2400000 & Error & Type & Filter & Reference \\
\hline V1137 Cas & 51473.568 & & P & V & IBVS 5570 \\
V1137 Cas & 53613.4050 & 0.0002 & S & R & IBVS 5741 \\
V1137 Cas & 53613.40344 & 0.0002 & S & R & OEJV 74 \\
V1137 Cas & 53615.45611 & 0.0003 & P & R & New - This study \\
V1137 Cas & 53746.48711 & 0.0009 & S & R & New - This study \\
\hline
\end{tabular}

Notes. Table is published in its entirety at the CDS. A portion is shown here for guidance regarding its form and content.

For all of the systems, the presence of the third light was tested. This new free parameter was only applied during the last step of the fitting process, because it is a very sensitive secondorder light curve parameter, which could only be conclusively detected in high quality LCs.

\section{Results}

The selection of targets for the present analysis was straightforward, and did not depend on where the star is located, nor how bright or faint it is. The criterion was based only whether the star had been previously studied, as our main aim was to enlarge the existing set of eclipsing binaries in the period - eccentricity diagram.

Another selection criterion was the data coverage of the particular binary. Only those systems with well-sampled phase light curve were included into our sample. This means that photometry should exist and especially that it should be adequately precise near both eclipses, so that their duration and depths could be recovered. Sometimes we had to use more data sources for this analysis (because in one dataset the coverage of minima is only poor).

The stars included in our sample can be divided into three groups according to their discoveries of photometric periodicity. Some have already been mentioned in the former ASAS database (Pojmanski 2002), a second group were serendipitous discoveries already mentioned in the literature (mainly the socalled CzeV catalogue, Skarka et al. 2017), and finally the third group consists of stars which have not yet been reported as eclipsing binaries, and this is for the first time that such stars have been identified as being an EEB system.

For the analysis we usually used some of the following available databases and surveys: ASAS (Pojmanski 2002), SuperWASP (Pollacco et al. 2006), NSVS (Woźniak et al. 2004), ASAS-SN (Shappee et al. 2014 and Kochanek et al. 2017), TAROT (Boër et al. 2001), INTEGRAL-OMC (Mas-Hesse et al. 2003), Bochum Galactic Disk Survey (Hackstein et al. 2015), CRTS (Drake et al. 2009), and sometimes also HipParcos (Perryman et al. 1997) when available for brighter sources. For one system (V1268 Tau) the photometry from the STEREO satellite (Kaiser et al. 2008 and Wraight et al. 2011) was also used. Our new photometry was obtained with various telescopes at different observatories (BOOTES in Spain, FRAM ${ }^{1}$ in Argentina, Danish 1.54-m telescope in Chile, $65-\mathrm{cm}$ telescope in Ondřejov, Czech Republic), but also with a nonnegligible contribution by several amateur observers with their small telescopes. For some of the systems we collected the data

\footnotetext{
1 FRAM (Ebr et al. 2014) telescope is part of the Pierre Auger Observatory (The Pierre Auger Collaboration 2015).
}

for more than ten years. The already known stars with published minima timings were checked and the data downloaded from the O-C gateway ${ }^{2}$ (Paschke \& Brát 2006).

The crucial parameters resulting from our light curve and apsidal motion fits are listed in Table A.2. For those stars where the apsidal motion is very slow, we have only indicated that the apsidal period is very long ( $>1000 \mathrm{yr}$ ), and that our data coverage is still too poor to facilitate a reliable analysis. This can clearly be seen in the Fig. A.1 with the $\mathrm{O}-\mathrm{C}$ diagrams. The parameters $H J D_{0}$ and $P$ stand for the average ephemerides representing the value $\mathrm{O}-\mathrm{C}=0$ in the $\mathrm{O}-\mathrm{C}$ diagrams, meaning that these are not suitable for planning the observations.

Concerning the presented solutions given in Table A.2, we still have to emphasize that these are still only the preliminary results, based on photometry only. The same applies for the errors of individual parameters given in Table A.2, which are based on the errors given by the PHOEBE program. Sometimes these errors are rather underestimated.

What definitely should be mentioned is that several systems have quite unrealistic parameters concerning their temperatures, radii, and luminosities. We are aware of the fact that the solution presented here (having $q=1.0$ ) is unlikely, or at least improbable. Hence, for these problematic cases we decided to use the approach for deriving the mass ratio from the luminosity ratio given in Graczyk (2003). This method uses the assumption that both components are located on the main sequence. However, using this approach we found out that for a few such cases even this method is not able to describe the observed data adequately and provide us with physically unreliable result. These are the cases like V1137 Cas, or CzeV 1279. It may be caused by the fact that the mass ratio derivation is based on the assumption of the main sequence components. For some others (such as e.g. NO Per, CzeV 364, SS TrA, V1301 Sco, V1344 her, or PS Vul) the components are probably giants, or some overluminous stars according to their effective temperatures. Only further, more detailed, study would be able to reveal their true nature. Because the number of systems is rather large, we cannot focus on all of the systems in detail, hence we would like to point out here only the most interesting targets.

Amongst the systems reported in this study, several systems were found to contain additional close visual components. These are: V1018 Cas, V1268 Tau, HD 55338, V611 Pup, V839 Cep, V922 Cep, and V389 And, respectively. For some of them a nonzero value of the third light was also detected during the light curve solution.

One system is a giant star, namely CD-33 2771, which merits special attention for future investigation. It has also the longest orbital period of about 200 days. Because this star has such a long period it would not be expected to show wide eclipses when it is not a giant star. According to our modelling, the primary component is probably of luminosity class III, while the secondary of subgiant class IV.

Also remarkable are systems where some possible additional variation in the $\mathrm{O}-\mathrm{C}$ diagram appears after subtraction of the apsidal motion term. These are mainly: V1137 Cas, KO Nor, V883 Sco, and V1301 Sco. These should be suitable for further observations to detect these changes, and to confirm the thirdbody hypothesis or other phenomena. See Fig. 1 for their $\mathrm{O}-\mathrm{C}$ diagrams after subtraction of the apsidal motion.

Amongst all of the studied systems, we have chosen those with given some spectral type estimates and derived the

\footnotetext{
2 See http://var2.astro.cz/ocgate/
} 

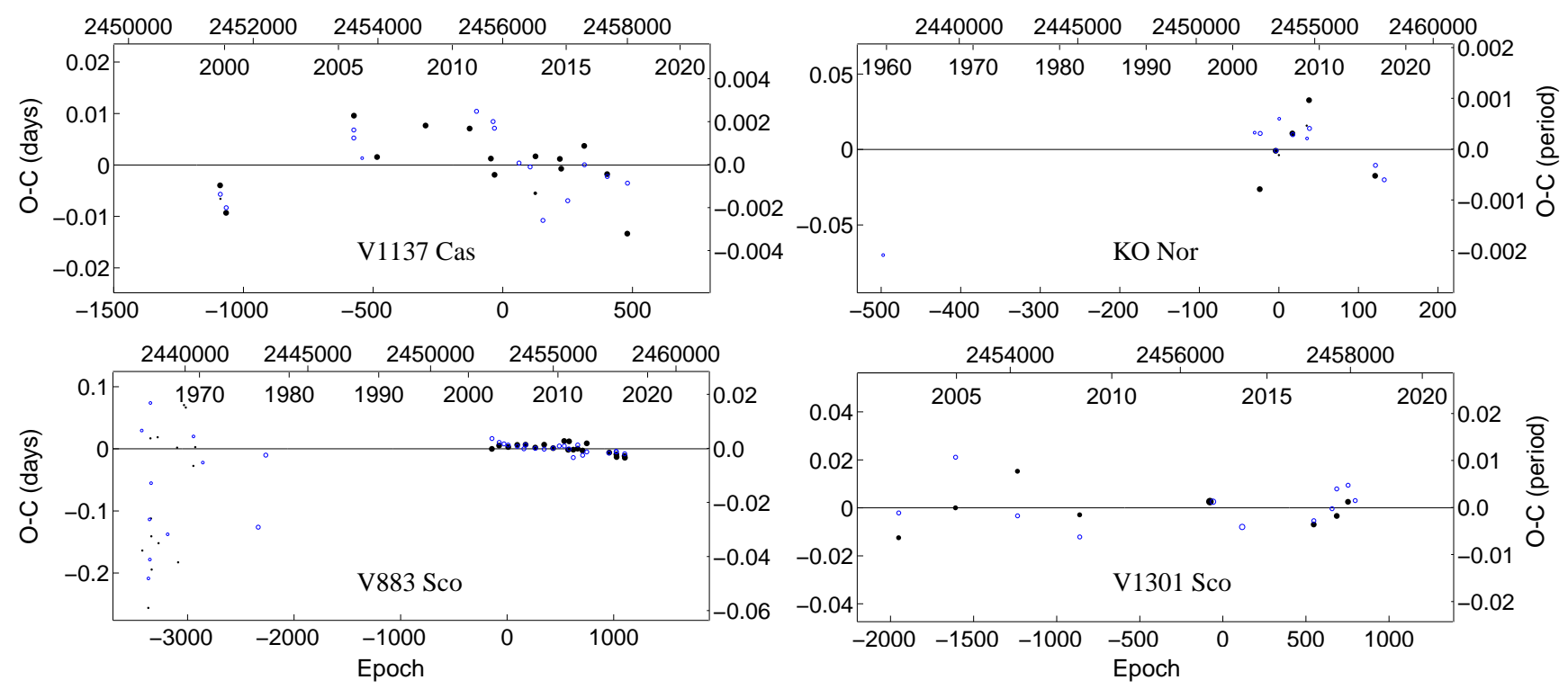

Fig. 1. O-C diagrams after subtraction of the apsidal motion term. These four systems are suspected for some additional variation of their minima times, hence deserve a special attention.

Table 2. Apsidal rotators with significant relativistic contribution.

\begin{tabular}{ll}
\hline \hline Star & $\dot{\omega}_{\text {rel }} / \dot{\omega}_{\text {total }}$ \\
\hline V1268 Tau & $59.9 \pm 35.0 \%$ \\
V437 Aur & $37.5 \pm 14.7 \%$ \\
HD 44093 & $52.8 \pm 18.4 \%$ \\
V611 Pup & $73.2 \pm 40.8 \%$ \\
HD 163735 & $22.3 \pm 15.7 \%$ \\
HD 313631 & $57.1 \pm 23.5 \%$ \\
V1344 Her & $55.0 \pm 42.8 \%$ \\
\hline
\end{tabular}

relativistic contribution to the total apsidal motion. This is viable only when we know the masses, in cases when the temperatures were only derived using the photometric indices the masses are too uncertain for any such analysis. Following the method by Giménez (1985) we derived the relativistic apsidal advance and for those systems with the largest contribution we gave their values in Table 2. As one can see, these values are affected by large errors due to the fact that the apsidal advance is very slow and only small fraction of the whole apsidal period is covered with data nowadays. These systems also deserve detailed study in the future.

Eccentric eclipsing binaries which have been studied quite frequently, and the known systems were also included in various catalogues of EEBs. The first one is for example, by Hegedüs (1988), later updated by Petrova \& Orlov (1999), while the most recent compilation of those stars is presented in Bulut \& Demircan (2007). Their catalogue contains a total of 108 systems with known eccentricities and apsidal motion periods. Therefore, our set of 54 new EEBs presents a significant contribution to the topic.

One can compare these two sets of stars and plot several interesting statistics. As can be seen in Fig. 2, our new systems have slightly later spectral types, and are also rather fainter than the original sample by Bulut \& Demircan (2007). This is caused by the fact that we mainly used the photometry from the various databases and automatic surveys, which are typically focused on stars fainter than $10 \mathrm{mag}$. On the other hand, the catalogue by
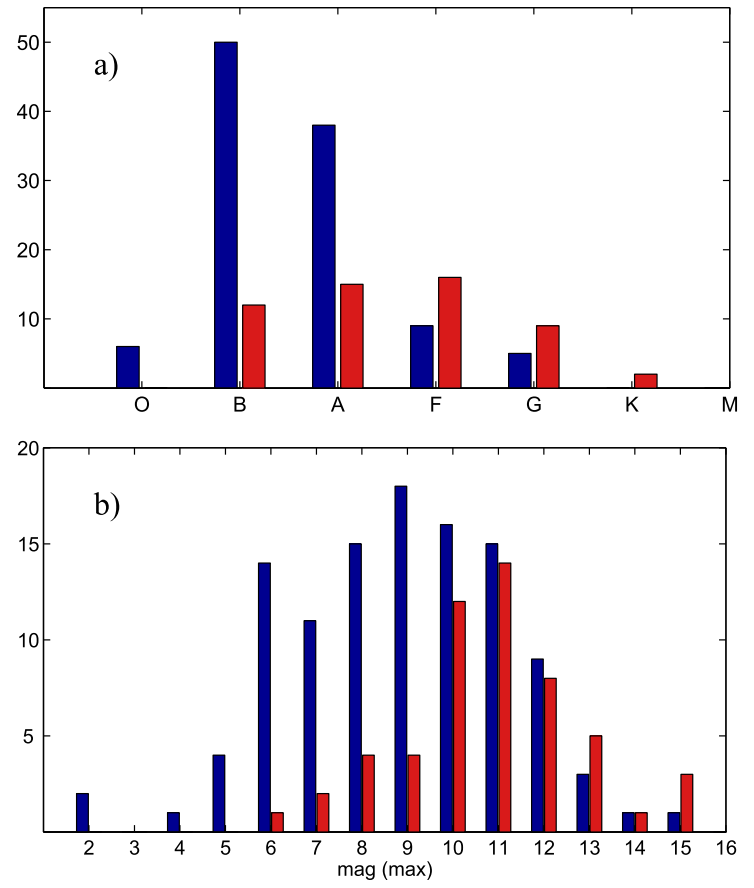

Fig. 2. Comparison of our sample of eccentric binaries (red) with the catalogue of eccentric systems by Bulut \& Demircan (2007) in blue. Upper plot: distribution of spectral types. Lower plot: apparent magnitudes of the systems.

Bulut \& Demircan (2007) presented all the well-known systems that have been studied for up to decades or even more, and that are sometimes as bright as naked-eye stars. The distribution of spectral types is a little more complicated to describe due to the fact that we do not know the interstellar reddening for most of these stars, and that the assumed spectral types were only derived on the basis of their photometric indices (i.e. a kind of lower limit). However, the stars having early spectral types have mostly been studied during the last century, and it is only during the last decades with the use of large telescopes did the focus of stellar astronomers shift to the more late and fainter systems. 


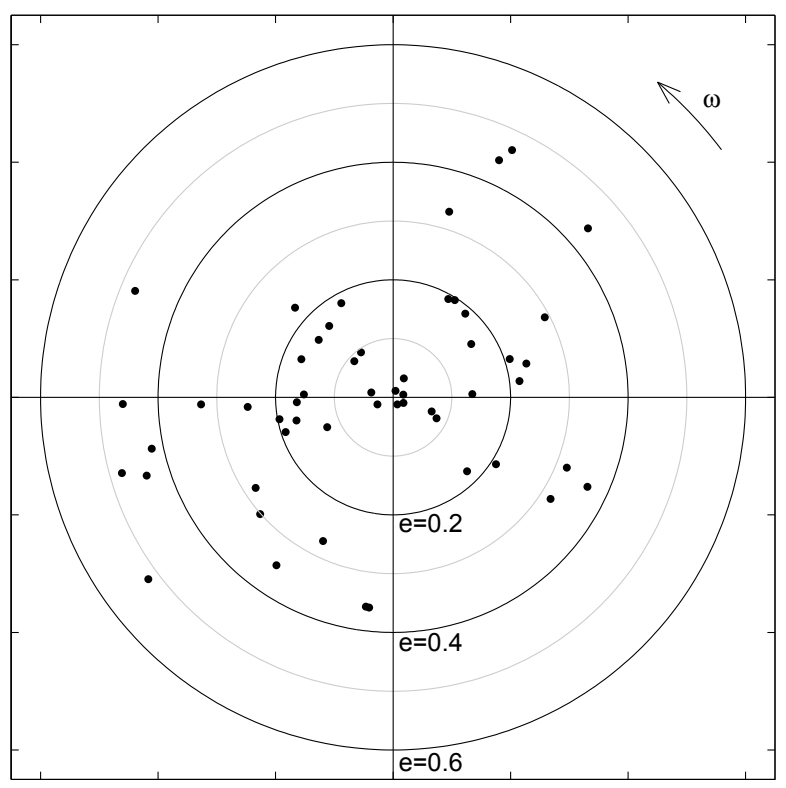

Fig. 3. Distribution of omega angles with respect to the eccentricity.

We also have more systems with longer orbital periods $(>30 \mathrm{~d})$ than appear in Bulut \& Demircan (2007).

We can also address the issue of selection effects for our sample of stars. These "serendipitous" discoveries should be more likely detected as eccentric when the two minima are displaced from their positions 0.0 and 0.5 in phase diagram, but the binaries which have $\omega$ close to $90^{\circ}$ or $270^{\circ}$ should be only rarely detected. This can more easily be done when the photometry is sufficiently precise and also when the eccentricity is higher (i.e. the durations of both eclipses are significantly different). Hence, we tried to test this distribution of $\omega$ versus eccentricity in our Fig. 3. One can see that the diagram is almost uniformly covered with data points, but the number of systems with higher eccentricity is still rather low (see Sect. 4).

\section{Period eccentricity relation}

The diagram which should be discussed in more detail is the period-eccentricity distribution of our systems. We plotted our results together with those already published before by Bulut $\&$ Demircan (2007) and also with the spectroscopic binaries from the SB9 catalogue by Pourbaix et al. (2004) in the Fig.4. As can be seen, orbital circularization plays a role in binaries with shorter periods (Raghavan et al. 2010, Duchêne \& Kraus 2013), while practically all systems that have periods below one day are already circular. Besides the plotted observed data we have also plotted several solid lines representing the limits of very close periastron approaches of both components (i.e. $1.5 \times R_{\star}$ ) when these likely collide with each other. These periastron distances were computed for different spectral types (B to $\mathrm{M}$ ) according to their typical radii and masses (see Zombeck 1990) with the assumption that both components are similar to each other (same masses and radii). As we can see, the sample of eclipsing apsidal motion systems has increased significantly with our new data set and should be very helpful in future when analysing the P-e diagram in detail.

However, we still have to be very careful when interpreting these results with our new data. For some systems a significant change of eccentricity could appear for the case when the star still has poor data coverage of both light curve and the $\mathrm{O}-\mathrm{C}$

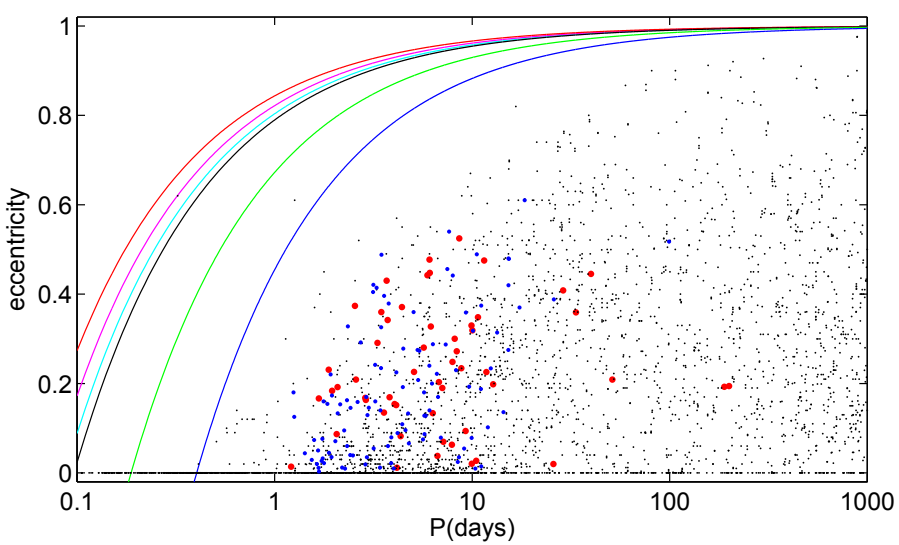

Fig. 4. Distribution of known eccentric systems in the periodeccentricity diagram. New data are plotted in red, systems from the catalogue by Bulut \& Demircan (2007) in blue, and spectroscopic binaries from the SB9 catalogue (Pourbaix et al. 2004) as small black points. See the text for detailed description of the solid lines.

diagram. The P-e diagram still lacks of such systems which have significant eccentricity close to its upper limit for a particular period. The system with the highest eccentricity from the catalogue by Bulut $\&$ Demircan (2007) is LV Her $(P=18.44 \mathrm{~d}, e=0.61)$, while some more eccentric eclipsing systems definitely exist in the catalogue of OGLE (Zasche, in prep.), and Kepler (Prša, priv. comm.).

\section{Discussion and conclusions}

We have derived the preliminary apsidal motion and light curve parameters for 54 Algol-type binaries. This is the first time any such analysis of such a large sample of eccentric eclipsing binaries has been studied in our Galaxy using different sources of photometry. Bringing together data from various databases and surveys has facilitated estimation of the long-term evolution of the orbit and the apsidal precession of our sample. Hong et al. (2016) is the only similar study of such a large sample of stars. The authors present 90 eclipsing binaries with apsidal motion. However, their study only used the OGLE III database (Graczyk et al. 2011) covering eight seasons. The advantage of our approach for the galactic targets is the fact that we also used archival photometry and the already published data reach back even to the 1930s in one case (SS TrA), hence the detected slow apsidal motion should be more conclusive.

We should also mention the difference between our sample and the one already published earlier by Bulut $\&$ Demircan (2007) - both in magnitude ranges as well as spectral types. The assumed spectral type and the distribution of both periods (orbital and apsidal motion) can also be studied as noted for example, by Hong et al. (2016). They presented a diagram showing that there is a possible relation between both periods and the masses of the components. However, they concluded that a much larger sample is needed for the final verdict, especially the longer-periodic binaries $(\mathrm{P}>6 \mathrm{~d})$ that also have longer apsidal motions. In our contribution to the topic more than $50 \%$ of our studied systems have periods longer than six days.

The sample of 54 systems presented in this study provides a good starting point for future dedicated observations and analyses of several more interesting systems, for example, these showing some suspicious additional variability of their orbital periods, these with rapid apsidal motion, or those containing close components. As good spectroscopic data (i.e. knowing the 
individual masses) is also available for them, one should be able to derive the internal structure constants or compute the relativistic contribution to the total apsidal motion rate.

Acknowledgements. GJW gratefully thanks the Leverhulme Trust for financial support through a Fellowship. We would like to thank the Pierre Auger Collaboration for the use of its facilities. The operation of the robotic telescope FRAM was supported by the EU grant GLORIA (No. 283783 in FP7-Capacities programme) and by the grants of the Ministry of Education of the Czech Republic (MSMT-CR LM2015038 and LTT18004). The data calibration and analysis related to FRAM telescope is supported by the Ministry of Education of the Czech Republic MSMT-CR CZ.02.1.01/0.0/0.0/16_013/0001402. The STEREO $\mathrm{HI}$ instrument featured in this study was developed by a collaboration that included the Rutherford Appleton Laboratory and the University of Birming ham, both in the United Kingdom, the Centre Spatial de Liege (CSL), Belgium, and the US Naval Research Laboratory (NRL), Washington DC, USA. The STEREO/SECCHI project is an international consortium of the Naval Research Laboratory (USA), Lockheed Martin Solar and Astrophysics Laboratory (USA), NASA Goddard Space Flight Center (USA), Rutherford Appleton Laboratory (UK), University of Birmingham (UK), Max-Planck-Institut fur Sonnensystemforschung (Germany), Centre Spatial de Liege (Belgium), Institut d'Optique Theorique et Appliquee (France) and Institut d'Astrophysique Spatiale (France) This paper makes use of data from the DR1 of the WASP data (Butters et al 2010) as provided by the WASP consortium, and the computing and storage facilities at the CERIT Scientific Cloud, reg. no. CZ.1.05/3.2.00/08.0144 which is operated by Masaryk University, Czech Republic. Work is based on the data from the OMC Archive at CAB (INTA-CSIC), pre-processed by ISDC. We thank the ASAS, NSVS, SuperWASP, OMC, and ASAS-SN teams for making all of the observations easily public available. This work was supported by the Czech Science Foundation grant no. GA15-02112S. Mr.Burkhardt from University of Heidelberg is also greatly acknowledged for sending us the old scanned publications. This research has made use of the SIMBAD database, operated at CDS, Strasbourg, France, and of NASA's Astrophysics Data System Bibliographic Services.

Note added in proof. A new study on eccentric eclipsing binaries by Kim et al. (2018) was published while this manuscript was in the final stages of publication. For five of the systems in common between Kim et al. (2018) and our paper, the results are slightly different. This is likely to be due to different methodologies and possibly also the different weightings used for the times of minima.

\section{References}

Alknis, A. 1958, Trudy Astrofiz. Lab. Riga, 7, 33

Boër, M., Atteia, J. L., Bringer, M., et al. 2001, A\&A, 378, 76

Borkovits, T., Hajdu, T., Sztakovics, J., et al. 2016, MNRAS, 455, 4136

Bulut, I., \& Demircan, O. 2007, MNRAS, 378, 179

Butters, O. W., West, R. G., Anderson, D. R., et al. 2010, A\&A, 520, L10

Drake, A. J., Djorgovski, S. G., Mahabal, A., et al. 2009, ApJ, 696, 870

Duchêne, G., \& Kraus, A. 2013, ARA\&A, 51, 269

Dyson, F. W. 1935, Second Greenwich Catalogue of Stars for 1925.0 (London)

Ebr, J., Janeček, P., Prouza, M., et al. 2014, Rev. Mex. Astron. Astrofis. Conf. Ser., 45,114

ESA 1997, ESA SP, 1200

Garrison, R. F., Hiltner, W. A., \& Schild, R. E. 1977, ApJS, 35, 111

Gimenez, A. 1985, ApJ, 297, 405

Giménez, A., \& García-Pelayo, J. M. 1983, Ap\&SS, 92, 203

Giménez, A., \& Bastero, M. 1995, Ap\&SS, 226, 99
Ginestet, N., \& Carquillat, J. M. 2002, ApJS, 143, 513

Graczyk, D. 2003, MNRAS, 342, 1334

Graczyk, D., Soszyński, I., Poleski, R., et al. 2011, Acta Astron., 61, 103

Hackstein, M., Fein, C., Haas, M., et al. 2015, Astron. Nachr., 336, 590

Hegedüs, T. 1988, Bulletin d'Information du Centre de Données Stellaires, 35, 15

Henden, A. A., Levine, S., Terrell, D., \& Welch, D. L. 2015, AAS Meeting Abstracts, 225, 336.16

Høg, E., Fabricius, C., Makarov, V. V., et al. 2000, A\&A, 355, L27

Hong, K., Lee, J. W., Kim, S.-L., Koo, J.-R., \& Lee, C.-U. 2016, MNRAS, 460, 650

Houk, N. 1978, Michigan catalogue of two-dimensional spectral types for the HD stars (Ann Arbor: Univ. of Michigan)

Houk, N. 1982, Michigan catalogue of two-dimensional spectral types for the HD stars (Ann Arbor: Univ. of Michigan), 3

Houk, N., \& Cowley, A. P. 1975, Michigan catalogue of two-dimensional spectral types for the HD stars (Ann Arbor: Univ. of Michigan), 1

Houk, N., \& Smith-Moore, M. 1988, Michigan catalogue of two-dimensional spectral types for the HD stars (Ann Arbor: Univ. of Michigan), 4

Houk, N., \& Swift, C. 1999, Michigan catalogue of two-dimensional spectral types for the HD stars(Ann Arbor: Univ. of Michigan), 5

Kaiser, M. L., Kucera, T. A., Davila, J. M., et al. 2008, Space Sci. Rev., 136, 5

Kim, C.-H., Kreiner, J. M., Zakrzewski, B., et al. 2018, ApJS, 235, 41

Kiminki, D. C., \& Kobulnicky, H. A. 2012, ApJ, 751, 4

Kochanek, C. S., Shappee, B. J., Stanek, K. Z., et al. 2017, PASP, 129, 104502

Mas-Hesse, J. M., Giménez, A., Culhane, J. L., et al. 2003, A\&A, 411, L261

Morgan, H. R. 1933, Pub. of the U.S. Naval Observatory Second Ser., 13

Nesterov, V. V., Kuzmin, A. V., Ashimbaeva, N. T., et al. 1995, A\&AS, 110, 367

Parihar, P., Messina, S., Bama, P., et al. 2009, MNRAS, 395, 593

Paschke, A., \& Brát, L. 2006, OEJV, 23, 13

Perryman, M. A. C., Lindegren, L., Kovalevsky, J., et al. 1997, A\&A, 323, L49

Petrova, A. V., \& Orlov, V. V. 1999, AJ, 117, 587

Pilecki, B., Fabrycky, D., \& Poleski, R. 2007, MNRAS, 378, 757

Pojmanski, G. 2002, AcA, 52, 397

Pollacco, D. L., Skillen, I., Collier Cameron, A., et al. 2006, PASP, 118, 1407

Pourbaix, D., Tokovinin, A. A., Batten, A. H., et al. 2004, A\&A, 424, 727

Prša, A., \& Zwitter, T. 2005, ApJ, 628, 426

Raghavan, D., McAlister, H. A., Henry, T. J., et al. 2010, ApJS, 190, 1

Rydström, B. A. 1978, A\&AS, 32, 25

Samus, N. N., Kazarovets, E. V., Durlevich, O. V., Kireeva, N. N., \& Pastukhova, E. N. 2017, Astron. Rep., 61, 80

Shappee, B. J., Prieto, J. L., Grupe, D., et al. 2014, ApJ, 788, 48

Skarka, M., Mašek, M., Brát, L., et al. 2017, OEJV, 185, 1

Skrutskie, M. F., Cutri, R. M., Stiening, R., et al. 2006, AJ, 131, 1163

Stephenson, C. B., \& Sanduleak, N. 1971, Pub. of the Warner \& Swasey Observatory, 1

Terrell, D., \& Wilson, R. E. 2005, Ap\&SS, 296, 221

The Pierre Auger Collaboration 2015, Nucl. Instrum. Methods Phys. Res. Sect. A Accel. Spectrom. Detect. Assoc. Equip., 798, 172

Tokovinin, A. 2008, MNRAS, 389, 925

Tucker, R. H., Buontempo, M. E., Gibbs, P., \& Swifte, R. H. D. 1983, Royal Greenwich Observatory Bulletins, 189

Wilson, R. E. 1979, ApJ, 234, 1054

Wilson, R. E., \& Devinney, E. J. 1971, ApJ, 166, 605

Woźniak, P. R., Vestrand, W. T., Akerlof, C. W., et al. 2004, AJ, 127, 2436

Wraight, K. T., White, G. J., Bewsher, D., \& Norton, A. J. 2011, MNRAS, 416 2477

Zasche, P., Wolf, M., Uhlař, R., \& Kučáková, H. 2014a, AJ, 147, 130

Zasche, P., Wolf, M., Vraštil, J., et al. 2014b, A\&A, 572, A71

Zombeck, M. V. 1990, Handbook of space astronomy and astrophysics (Cambridge: University Press), 2nd ed. 


\section{Appendix A: Additional material}

Table A.1. Relevant information for the analysed systems.

\begin{tabular}{|c|c|c|c|c|c|c|c|}
\hline $\begin{array}{l}\text { System } \\
\text { name }\end{array}$ & Other ID & RA & $\mathrm{DE}$ & $\begin{array}{c}V_{\max }^{A} \\
(\mathrm{mag})\end{array}$ & $\begin{array}{c}(J-H)^{B} \\
(\mathrm{mag})\end{array}$ & $\begin{array}{c}(B-V)^{A} \\
(\mathrm{mag})\end{array}$ & Sp.Type ${ }^{C}$ \\
\hline V1137 Cas & GSC 04297-01664 & $01^{\mathrm{h}} 34^{\mathrm{m}} 53^{\mathrm{s}} .930$ & $+67^{\circ} 38^{\prime} 15^{\prime \prime} .00$ & $11^{\mathrm{m}} \cdot 81$ & 0.255 & 0.80 & \\
\hline CR Per & AN 16.1940 & $02^{\mathrm{h}} 09^{\mathrm{m}} 52^{\mathrm{s}} .170$ & $+57^{\circ} 54^{\prime} 32^{\prime \prime} .28$ & $12^{\mathrm{m}} \cdot 02$ & 0.100 & 0.47 & \\
\hline CzeV 662 & GSC 03691-00735 & $02^{\mathrm{h}} 36^{\mathrm{m}} 48^{\mathrm{s}} .615$ & $+56^{\circ} 04^{\prime} 11^{\prime \prime} .92$ & $12^{\mathrm{m}} \cdot 96$ & 0.077 & 0.36 & \\
\hline $\mathrm{CzeV} 701$ & TYC 3700-608-1 & $02^{\mathrm{h}} 40^{\mathrm{m}} 23^{\mathrm{s}} .897$ & $+54^{\circ} 14^{\prime} 34^{\prime \prime} .92$ & $10^{\mathrm{m}} \cdot 81$ & 0.254 & 0.52 & \\
\hline CzeV 688 & GSC 03708-01145 & $02^{\mathrm{h}} 47^{\mathrm{m}} 20^{\mathrm{s}} .119$ & $+56^{\circ} 15^{\prime} 05^{\prime \prime} .63$ & $13^{\mathrm{m}} \cdot 12$ & 0.254 & 1.01 & \\
\hline V1018 Cas & GSC 04048-00934 & $03^{\mathrm{h}} 01^{\mathrm{m}} 19^{\mathrm{s}} .374$ & $+60^{\circ} 34^{\prime} 20^{\prime \prime} .24$ & $10^{\mathrm{m}} \cdot 24$ & 0.099 & 0.72 & B2III [1] \\
\hline V1268 Tau & HIP 17168 & $03^{\mathrm{h}} 40^{\mathrm{m}} 38^{\mathrm{s}} .770$ & $+28^{\circ} 46^{\prime} 24^{\prime \prime} .00$ & $7^{m} \cdot 38$ & 0.050 & 0.15 & $\mathrm{~A} 0[2]$ \\
\hline NOPer & $\mathrm{SV} * \mathrm{SON} 8551$ & $04^{\mathrm{h}} 15^{\mathrm{m}} 41^{\mathrm{s}} .820$ & $+48^{\circ} 40^{\prime} 42^{\prime \prime} .10$ & $12^{\mathrm{m}} \cdot 21$ & 0.216 & 0.89 & \\
\hline CzeV 1279 & UCAC4 093-005674 & $04^{\mathrm{h}} 50^{\mathrm{m}} 58^{\mathrm{s}} .786$ & $-71^{\circ} 31^{\prime} 49^{\prime \prime} .35$ & $11^{\mathrm{m}} \cdot 07$ & 0.285 & 0.38 & \\
\hline DT Cam & HIP 24390 & $05^{\mathrm{h}} 13^{\mathrm{m}} 57^{\mathrm{s}} .689$ & $+56^{\circ} 30^{\prime} 28^{\prime \prime} .63$ & $8^{\mathrm{m}} \cdot 19$ & 0.054 & 0.20 & $\mathrm{~A} 2[2]$ \\
\hline UCAC4 609-022916 & 2MASS J05455225+3142200 & $05^{\mathrm{h}} 45^{\mathrm{m}} 52^{\mathrm{s}} .250$ & $+31^{\circ} 42^{\prime} 20^{\prime \prime} .00$ & $14^{\mathrm{m}} \cdot 83$ & 0.162 & 0.52 & \\
\hline V409 Cam & TYC 4524-1856-1 & $05^{\mathrm{h}} 46^{\mathrm{m}} 43^{\mathrm{s}} .904$ & $+75^{\circ} 20^{\prime} 56^{\prime \prime} .51$ & $10^{\mathrm{m}} \cdot 73$ & 0.136 & 0.42 & \\
\hline CzeV 1144 & UCAC4 602-024605 & $05^{\mathrm{h}} 48^{\mathrm{m}} 07^{\mathrm{s}} .920$ & $+30^{\circ} 13^{\prime} 19^{\prime \prime} .10$ & $14^{\mathrm{m}} .59$ & 0.158 & 0.46 & \\
\hline V437 Aur & HIP 27469 & $05^{\mathrm{h}} 49^{\mathrm{m}} 03^{\mathrm{s}} .060$ & $+54^{\circ} 01^{\prime} 57^{\prime \prime} .03$ & $8^{\mathrm{m}} \cdot 47$ & -0.016 & 0.03 & B9 [3] \\
\hline CzeV 364 & GSC $02405-01470$ & $05^{\mathrm{h}} 49^{\mathrm{m}} 40^{\mathrm{s}} .700$ & $+30^{\circ} 25^{\prime} 00^{\prime \prime} .70$ & $13^{\mathrm{m}} .95$ & 0.236 & 0.59 & \\
\hline $\mathrm{CzeV} 464$ & USNO-A2.0 1200-03882057 & $05^{\mathrm{h}} 50^{\mathrm{m}} 11^{\mathrm{s}} .541$ & $+31^{\circ} 19^{\prime} 40^{\prime \prime} .34$ & $15^{\mathrm{m}} \cdot 12$ & 0.103 & & \\
\hline TYC 3750-599-1 & GSC 03750-00599 & $05^{\mathrm{h}} 50^{\mathrm{m}} 52^{\mathrm{s}} .961$ & $+53^{\circ} 58^{\prime} 23^{\prime \prime} .60$ & $10^{\mathrm{m}} \cdot 60$ & -0.072 & 0.08 & \\
\hline TYC 729-1545-1 & GSC 00729-01545 & $06^{\mathrm{h}} 07^{\mathrm{m}} 18^{\mathrm{s}} .601$ & $+13^{\circ} 31^{\prime} 45^{\prime \prime} .93$ & $9^{\mathrm{m}} \cdot 26$ & 0.105 & 0.29 & \\
\hline CD-332771 & TYC 7076-1598-1 & $06^{\mathrm{h}} 10^{\mathrm{m}} 16^{\mathrm{s}} .479$ & $-33^{\circ} 21^{\prime} 20^{\prime \prime} .88$ & $9^{\mathrm{m}} \cdot 78$ & 0.724 & 1.47 & K5III+K5V [4] \\
\hline HD 44093 & GSC 00140-01064 & $06^{\mathrm{h}} 20^{\mathrm{m}} 04^{\mathrm{s}} .841$ & $+04^{\circ} 54^{\prime} 44^{\prime \prime} .60$ & $9^{\mathrm{m}} \cdot 25$ & -0.043 & 0.09 & B8/9V [5] \\
\hline TYC 5378-1590-1 & GSC 05378-01590 & $06^{\mathrm{h}} 45^{\mathrm{m}} 43^{\mathrm{s}} .953$ & $-08^{\circ} 50^{\prime} 35^{\prime \prime} .50$ & $10^{\mathrm{m}} \cdot 93$ & -0.029 & -0.02 & \\
\hline HD 55338 & TYC 4823-2213-1 & $07^{\mathrm{h}} 12^{\mathrm{m}} 20^{\mathrm{s}} .843$ & $-05^{\circ} 25^{\prime} 53^{\prime \prime} .94$ & $9^{\mathrm{m}} \cdot 54$ & 0.034 & 0.11 & A1IV/V [5] \\
\hline RW CMi & AN 128.1929 & $07^{\mathrm{h}} 22^{\mathrm{m}} 22^{\mathrm{s}} .590$ & $+02^{\circ} 26^{\prime} 22^{\prime \prime} .30$ & $12^{\mathrm{m}} \cdot 87$ & 0.139 & 0.39 & \\
\hline V611 Pup & HD 62589 & $07^{\mathrm{h}} 44^{\mathrm{m}} 06^{\mathrm{s}} .063$ & $-16^{\circ} 55^{\prime} 57^{\prime \prime} .95$ & $8^{\mathrm{m}} \cdot 09$ & -0.140 & -0.09 & B3III [6] \\
\hline CzeV 1283 & HD 68304 & $08^{\mathrm{h}} 09^{\mathrm{m}} 51^{\mathrm{s}} .160$ & $-45^{\circ} 37^{\prime} 50^{\prime \prime} .69$ & $10^{\mathrm{m}} \cdot 00$ & 0.146 & 0.40 & FoIV/V [11] \\
\hline TYC 7126-2416-1 & GSC 07126-02416 & $08^{\mathrm{h}} 16^{\mathrm{m}} 30^{\mathrm{s}} .996$ & $-33^{\circ} 14^{\prime} 38^{\prime \prime} .31$ & $10^{\mathrm{m}} \cdot 37$ & 0.483 & 0.93 & \\
\hline CzeV 1183 & 2MASS 08283756-4351041 & $08^{\mathrm{h}} 28^{\mathrm{m}} 37^{\mathrm{s}} .562$ & $-43^{\circ} 51^{\prime} 04^{\prime \prime} .11$ & $12^{\mathrm{m}} \cdot 43$ & 0.251 & 0.77 & \\
\hline DK Pyx & HIP 41980 & $08^{\mathrm{h}} 33^{\mathrm{m}} 24^{\mathrm{s}} .062$ & $-34^{\circ} 38^{\prime} 55^{\prime \prime} .31$ & $7^{\mathrm{m}} .84$ & -0.114 & -0.10 & B3III [7] \\
\hline PS UMa & TYC 4375-1733-1 & $08^{\mathrm{h}} 56^{\mathrm{m}} 46^{\mathrm{s}} .479$ & $+69^{\circ} 40^{\prime} 32^{\prime \prime} .12$ & $12^{\mathrm{m}} \cdot 45$ & 0.311 & 0.54 & \\
\hline HD 87803 & HIP 49354 & $10^{\mathrm{h}} 04^{\mathrm{m}} 31^{\mathrm{s}} .513$ & $-69^{\circ} 21^{\prime} 20^{\prime \prime} .27$ & $9^{\mathrm{m}} \cdot 51$ & 0.013 & 0.06 & B9.5V [8] \\
\hline TYC 8603-723-1 & GSC 08603-00723 & $10^{\mathrm{h}} 06^{\mathrm{m}} 25^{\mathrm{s}} .330$ & $-55^{\circ} 00^{\prime} 44^{\prime \prime} .60$ & $11^{\mathrm{m}} \cdot 38$ & -0.027 & 0.08 & \\
\hline HD 306001 & GSC 08958-03048 & $11^{\mathrm{h}} 06^{\mathrm{m}} 07^{\mathrm{s}} .882$ & $-61^{\circ} 09^{\prime} 09^{\prime \prime} .25$ & $9^{\mathrm{m}} \cdot 58$ & -0.071 & -0.09 & B5 [9] \\
\hline TYC 8217-789-1 & GSC 08217-00789 & $11^{\mathrm{h}} 15^{\mathrm{m}} 06^{\mathrm{s}} .983$ & $-48^{\circ} 15^{\prime} 33^{\prime \prime} .44$ & $11^{\mathrm{m}} \cdot 28$ & 0.186 & 0.65 & \\
\hline TYC 9432-1633-1 & 2MASS J14444107-7721530 & $14^{\mathrm{h}} 44^{\mathrm{m}} 41^{\mathrm{s}} .077$ & $-77^{\circ} 21^{\prime} 53^{\prime \prime} .02$ & $11^{\mathrm{m}} \cdot 70$ & 0.290 & 0.69 & \\
\hline SS TrA & GSC 09022-01335 & $15^{\mathrm{h}} 39^{\mathrm{m}} 21^{\mathrm{s}} .650$ & $-60^{\circ} 53^{\prime} 38^{\prime \prime} .30$ & $11^{\mathrm{m}} \cdot 02$ & 0.106 & 0.27 & \\
\hline KO Nor & TYC 8719-163-1 & $16^{\mathrm{h}} 25^{\mathrm{m}} 59^{\mathrm{s}} .508$ & $-56^{\circ} 55^{\prime} 03^{\prime \prime} .97$ & $10^{\mathrm{m}} \cdot 85$ & 0.082 & 0.62 & \\
\hline V883 Sco & HD 152901 & $16^{\mathrm{h}} 57^{\mathrm{m}} 52^{\mathrm{s}} .443$ & $-37^{\circ} 59^{\prime} 47^{\prime \prime} .57$ & $7^{\mathrm{m}} \cdot 04$ & 0.013 & 0.05 & $\mathrm{~B} 2 \mathrm{~V}[10]$ \\
\hline V1301 Sco & GSC 07368-01457 & $17^{\mathrm{h}} 05^{\mathrm{m}} 18^{\mathrm{s}} .635$ & $-34^{\circ} 56^{\prime} 00^{\prime \prime} .71$ & $13^{\mathrm{m}} .03$ & 0.228 & 0.68 & \\
\hline HD 158801 & TYC 7896-1604-1 & $17^{\mathrm{h}} 33^{\mathrm{m}} 19^{\mathrm{s}} .034$ & $-43^{\circ} 15^{\prime} 01^{\prime \prime} .40$ & $9^{\mathrm{m}} \cdot 54$ & 0.060 & 0.27 & A5/7II [11] \\
\hline TYC 6258-1011-1 & 2MASS J17533294-2031094 & $17^{\mathrm{h}} 53^{\mathrm{m}} 32^{\mathrm{s}} .945$ & $-20^{\circ} 31^{\prime} 09^{\prime \prime} .53$ & $12^{\mathrm{m}} \cdot 08$ & 0.182 & 0.48 & \\
\hline HD 163735 & TYC 5095-296-1 & $17^{\mathrm{h}} 57^{\mathrm{m}} 54^{\mathrm{s}} .027$ & $-05^{\circ} 41^{\prime} 10^{\prime \prime} .15$ & $9^{\mathrm{m}} \cdot 62$ & 0.251 & 0.54 & F3V [5] \\
\hline HD 313631 & TYC 6842-1455-1 & $18^{\mathrm{h}} 00^{\mathrm{m}} 10^{\mathrm{s}} .195$ & $-23^{\circ} 53^{\prime} 46^{\prime \prime} .10$ & $10^{\mathrm{m}} \cdot 41$ & 0.170 & 0.58 & OB [12] \\
\hline HD 164610 & GSC 07899-00130 & $18^{\mathrm{h}} 03^{\mathrm{m}} 39^{\mathrm{s}} .099$ & $-37^{\circ} 43^{\prime} 47^{\prime \prime} .68$ & $8^{\mathrm{m}} \cdot 64$ & 0.052 & 0.22 & A1mA8-A8 [7] \\
\hline V1344 Her & HD 348698 & $18^{\mathrm{h}} 27^{\mathrm{m}} 18^{\mathrm{s}} .443$ & $+19^{\circ} 08^{\prime} 33^{\prime \prime} .32$ & $11^{\mathrm{m}} \cdot 69$ & 0.228 & 0.27 & G0 [9] \\
\hline HD 170749 & TYC 7398-2681-1 & $18^{\mathrm{h}} 32^{\mathrm{m}} 34^{\mathrm{s}} .765$ & $-33^{\circ} 14^{\prime} 40^{\prime \prime} .85$ & $9^{\mathrm{m}} \cdot 94$ & 0.060 & 0.17 & $\mathrm{~A} 0 / 1 \mathrm{~V}[7]$ \\
\hline TYC 8378-252-1 & GSC 08378-00252 & $18^{\mathrm{h}} 59^{\mathrm{m}} 51^{\mathrm{s}} .282$ & $-47^{\circ} 11^{\prime} 47^{\prime \prime} .75$ & $11^{\mathrm{m}} \cdot 03$ & 0.168 & 0.58 & \\
\hline TYC 6303-308-1 & 2MASS J19393409-1739553 & $19^{\mathrm{h}} 39^{\mathrm{m}} 34^{\mathrm{s}} .096$ & $-17^{\circ} 39^{\prime} 55^{\prime \prime} .45$ & $11^{\mathrm{m}} \cdot 31$ & 0.110 & 0.37 & \\
\hline PS Vul & HIP 9709 & $19^{\mathrm{h}} 43^{\mathrm{m}} 55^{\mathrm{s}} .974$ & $+27^{\circ} 08^{\prime} 07^{\prime \prime} .43$ & $6^{\mathrm{m}} \cdot 46$ & 0.768 & 1.02 & $\mathrm{~K} 3 \mathrm{II}+\mathrm{B} 6[13]$ \\
\hline V839 Cep & TYC 3964-741-1 & $21^{\mathrm{h}} 03^{\mathrm{m}} 31^{\mathrm{s}} .714$ & $+59^{\circ} 25^{\prime} 50^{\prime \prime} .41$ & $9^{\mathrm{m}} \cdot 73$ & 0.055 & 0.36 & B8 [14] \\
\hline TYC 5195-11-1 & 2MASS J21264316-0031104 & $21^{\mathrm{h}} 26^{\mathrm{m}} 43^{\mathrm{s}} .166$ & $-00^{\circ} 31^{\prime} 10^{\prime \prime} .53$ & $11^{\mathrm{m}} \cdot 32$ & 0.233 & 0.49 & \\
\hline TYC 2712-1201-1 & GSC 2712-1201 & $21^{\mathrm{h}} 34^{\mathrm{m}} 57^{\mathrm{s}} .620$ & $+35^{\circ} 12^{\prime} 51^{\prime \prime} .46$ & $10^{\mathrm{m}} \cdot 70$ & -0.015 & -0.01 & \\
\hline UCAC4 585-123180 & NSVS 8774343 & $21^{\mathrm{h}} 39^{\mathrm{m}} 21^{\mathrm{s}} .030$ & $+26^{\circ} 52^{\prime} 36^{\prime \prime} .70$ & $12^{\mathrm{m}} \cdot 88$ & 0.264 & 0.57 & \\
\hline V922 Cep & TYC 4481-230-1 & $23^{\mathrm{h}} 01^{\mathrm{m}} 39^{\mathrm{s}} .222$ & $+69^{\circ} 42^{\prime} 44^{\prime \prime} .96$ & $11^{\mathrm{m}} 39$ & 0.137 & 0.29 & \\
\hline V389 And & HIP 116153 & $23^{\mathrm{h}} 32^{\mathrm{m}} 01^{\mathrm{s}} .312$ & $+43^{\circ} 49^{\prime} 20^{\prime \prime} .49$ & $8^{\mathrm{m}} \cdot 56$ & -0.031 & 0.19 & A0 [15] \\
\hline
\end{tabular}

Notes. (A) - value based on APASS (Henden et al. 2015) or Tycho catalogue Høg et al. (2000), (B) - 2MASS catalogue, Skrutskie et al. (2006), (C) - Various published papers.

References. [1] - Rydström (1978), [2] - Tucker et al. (1983), [3] - Morgan (1933), [4] - Parihar et al. (2009), [5] - Houk \& Swift (1999), [6] Houk \& Smith-Moore (1988), [7] - Houk (1982), [8] - Houk \& Cowley (1975), [9] - Nesterov et al. (1995), [10] - Garrison et al. (1977), [11] Houk (1978), [12] - Stephenson \& Sanduleak (1971), [13] - Ginestet \& Carquillat (2002), [14] - Alknis (1958), [15] - Dyson (1935). 


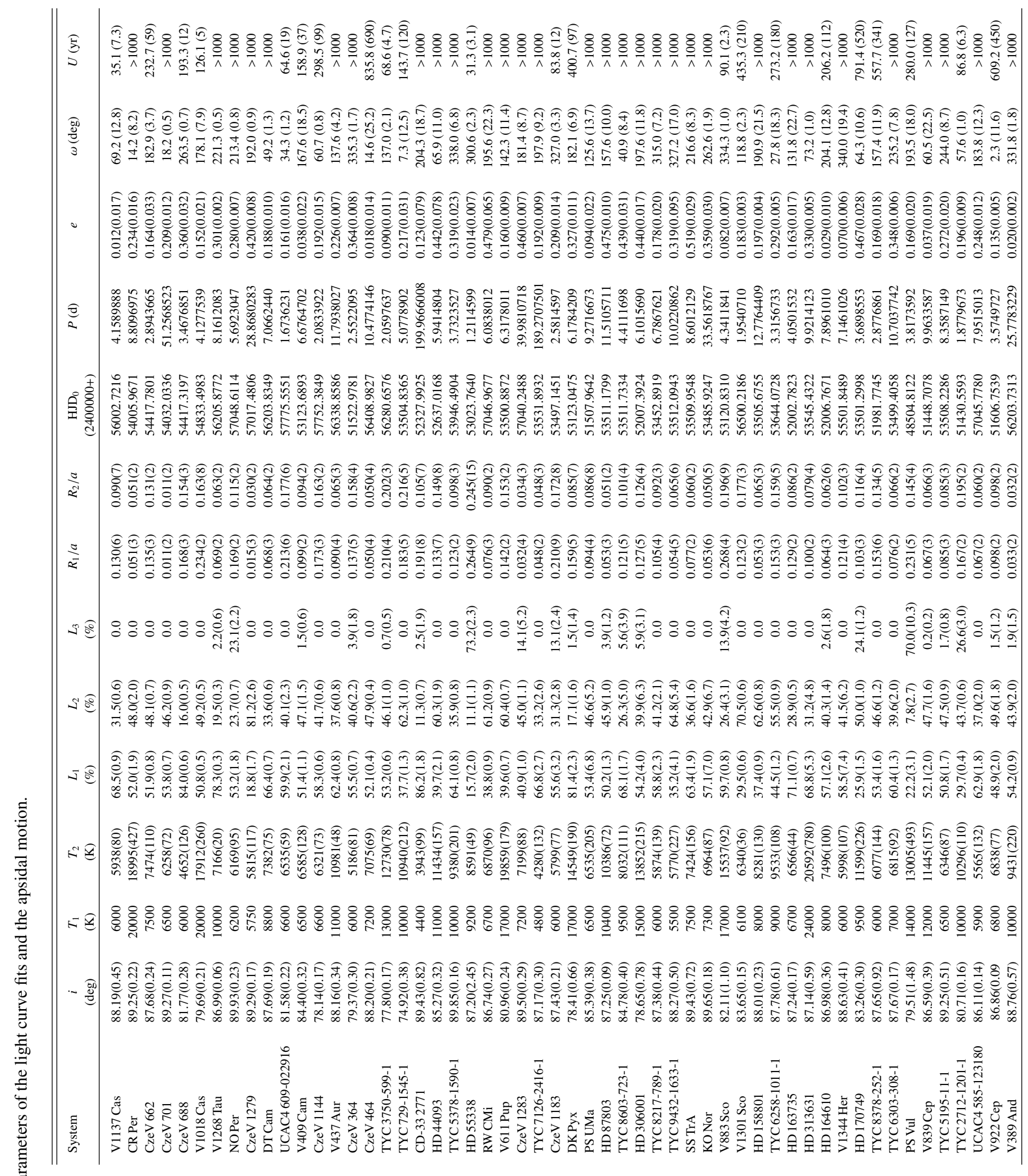


P. Zasche et al.: The first study of 54 eccentric eclipsing binaries
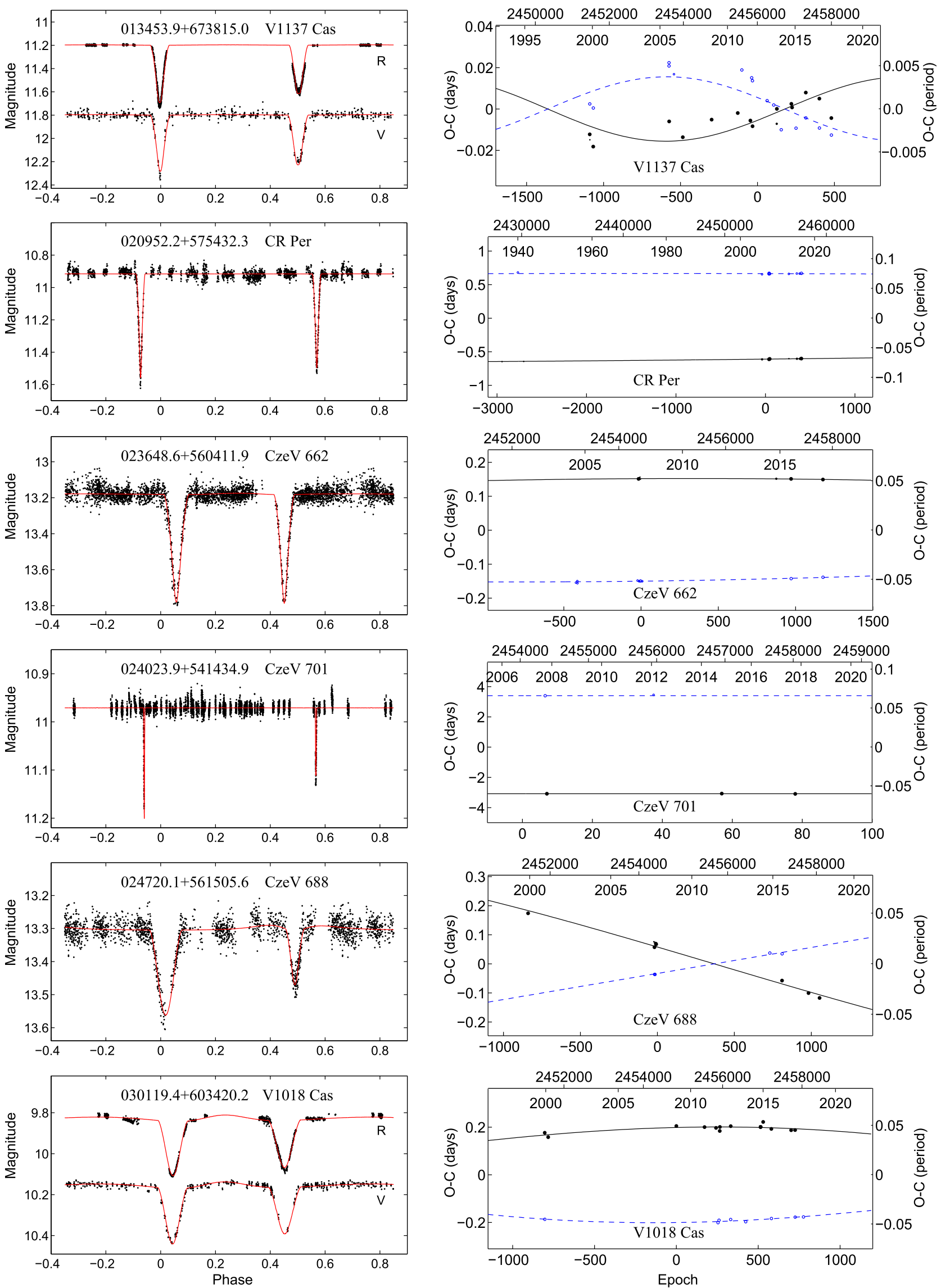

Fig. A.1. Light curves and $\mathrm{O}-\mathrm{C}$ diagrams of the analysed systems. The small letters denote the individual filters for the particular light curve (standard notation, while "C" stands for unfiltered data and " $\mathrm{S}$ " indicates the special SuperWASP filter). For the O-C diagrams the full dots stand for the primary minima (as well as the solid line), while the open circles represent the secondary minima (and the dashed curve). 
A\&A 619, A85 (2018)
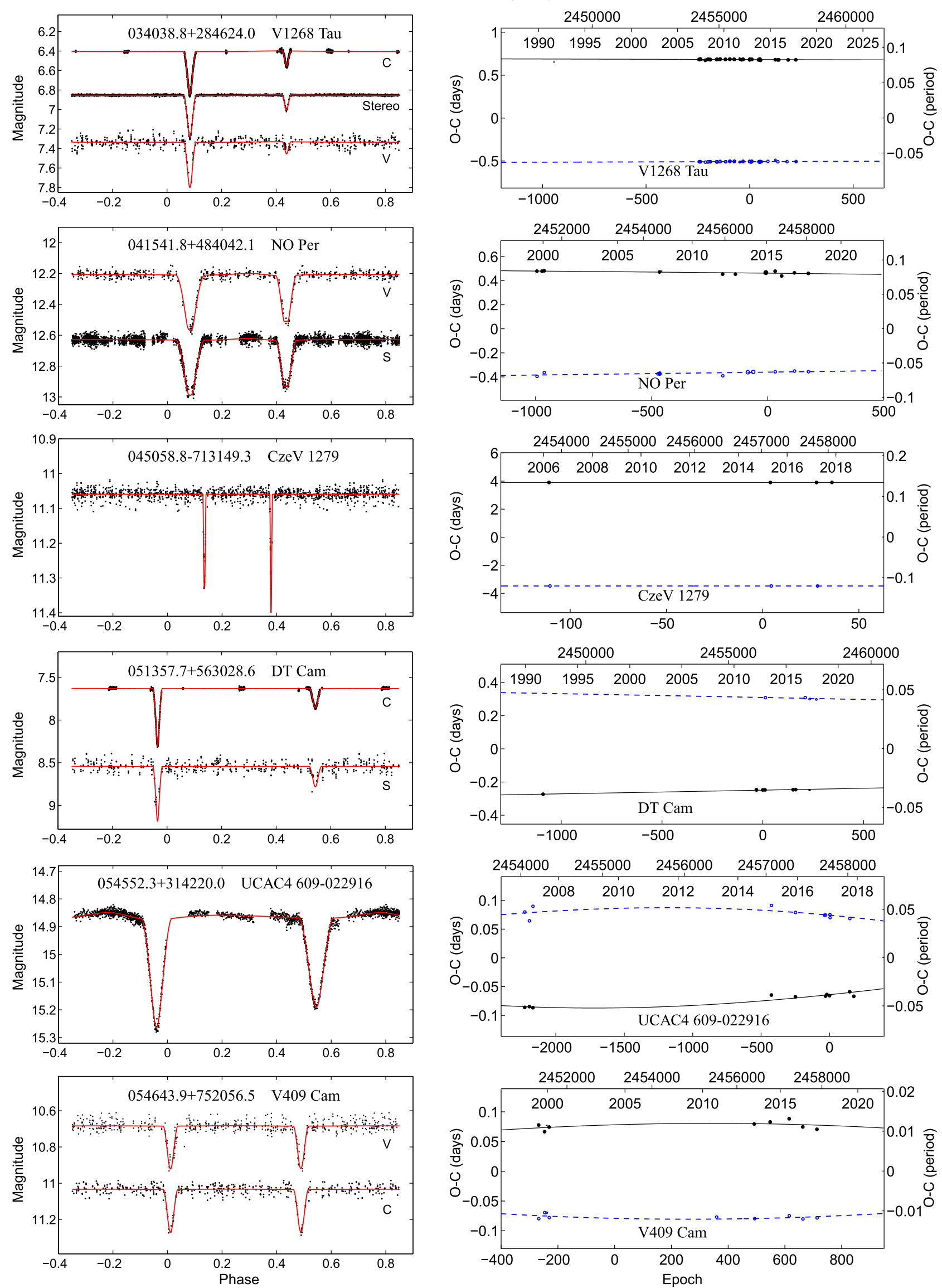

Fig. A.1. continued. 
P. Zasche et al.: The first study of 54 eccentric eclipsing binaries
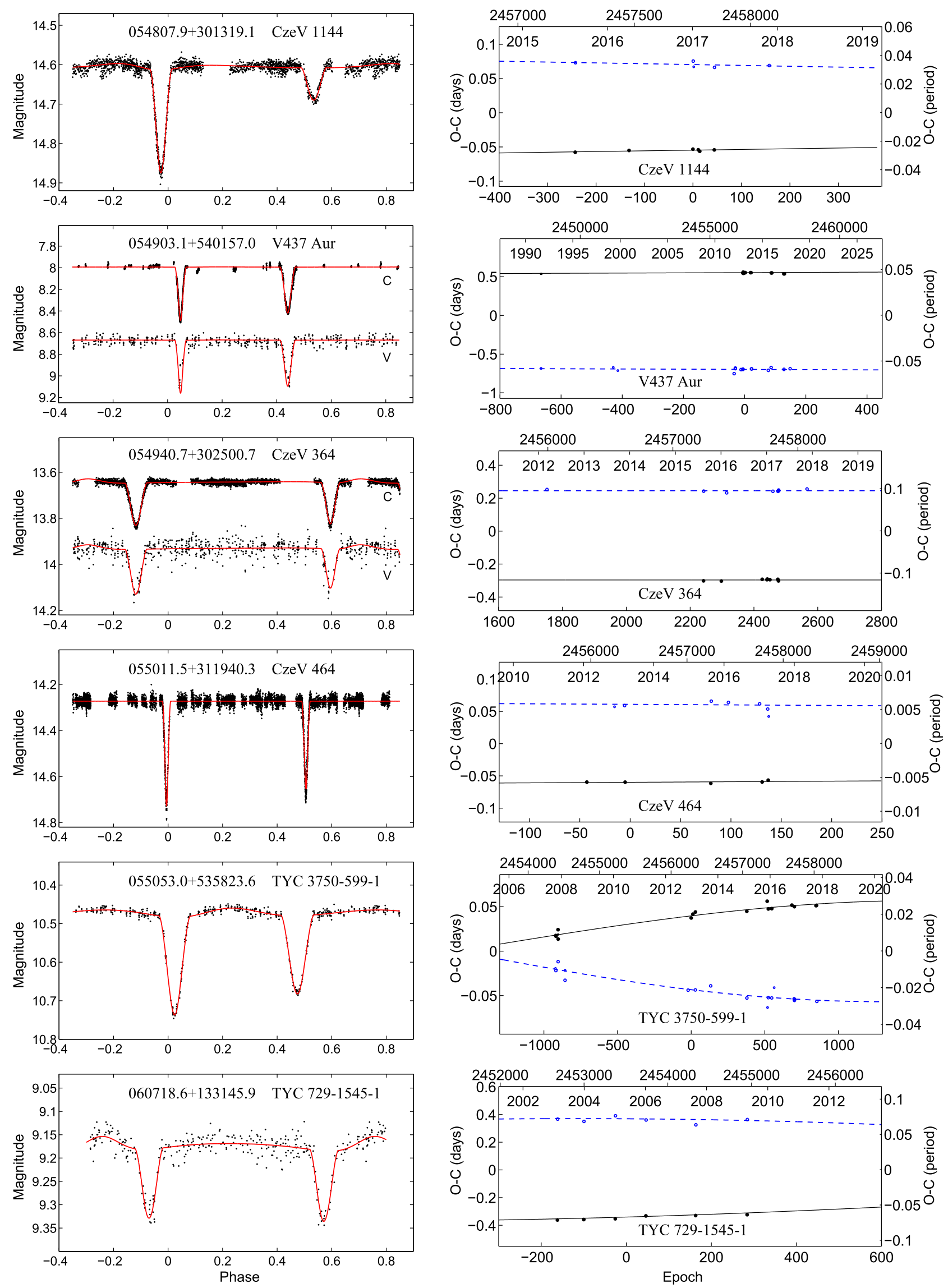

Fig. A.1. continued. 

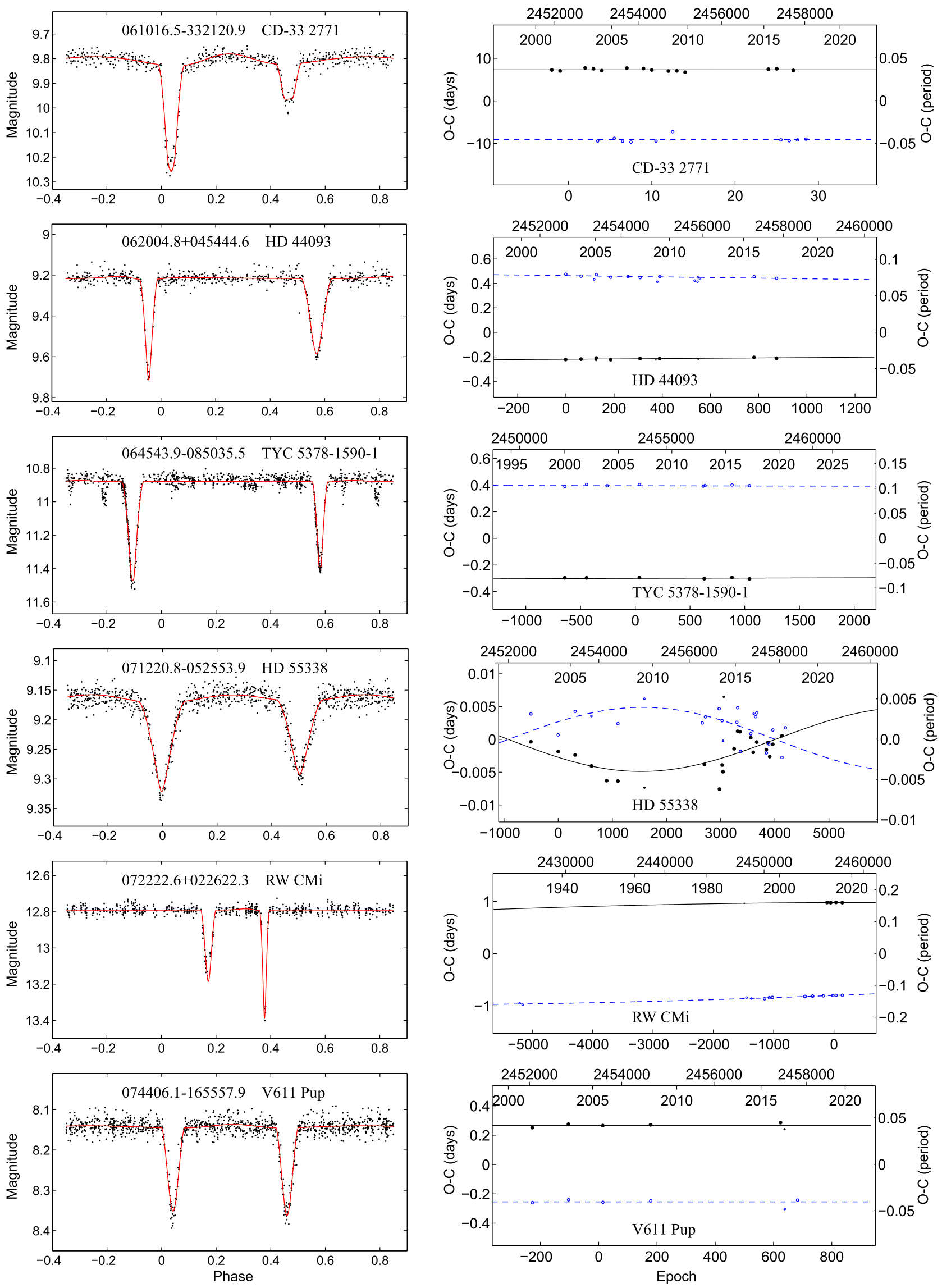

Fig. A.1. continued. 
P. Zasche et al.: The first study of 54 eccentric eclipsing binaries
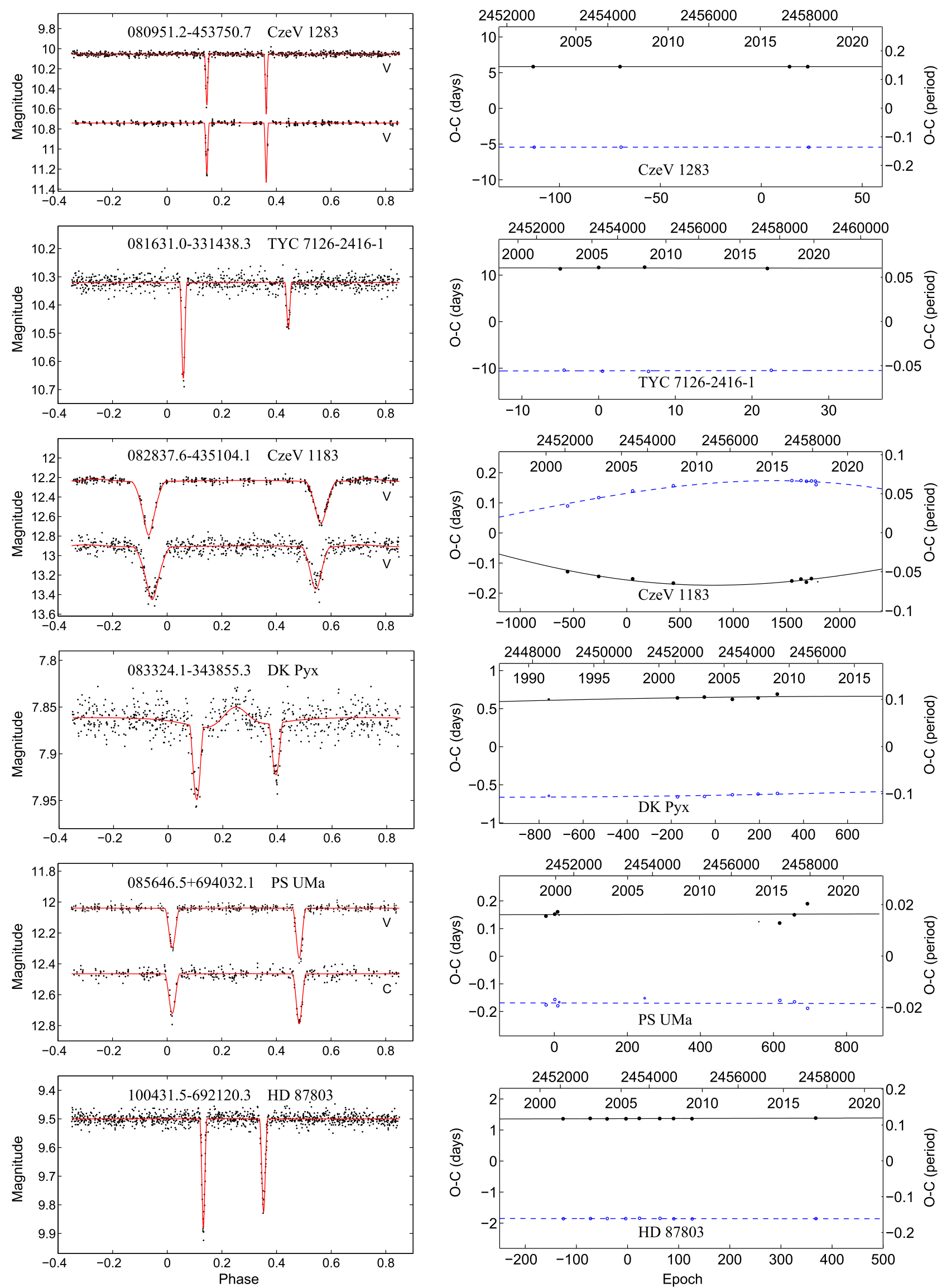

Fig. A.1. continued. 
A\&A 619, A85 (2018)
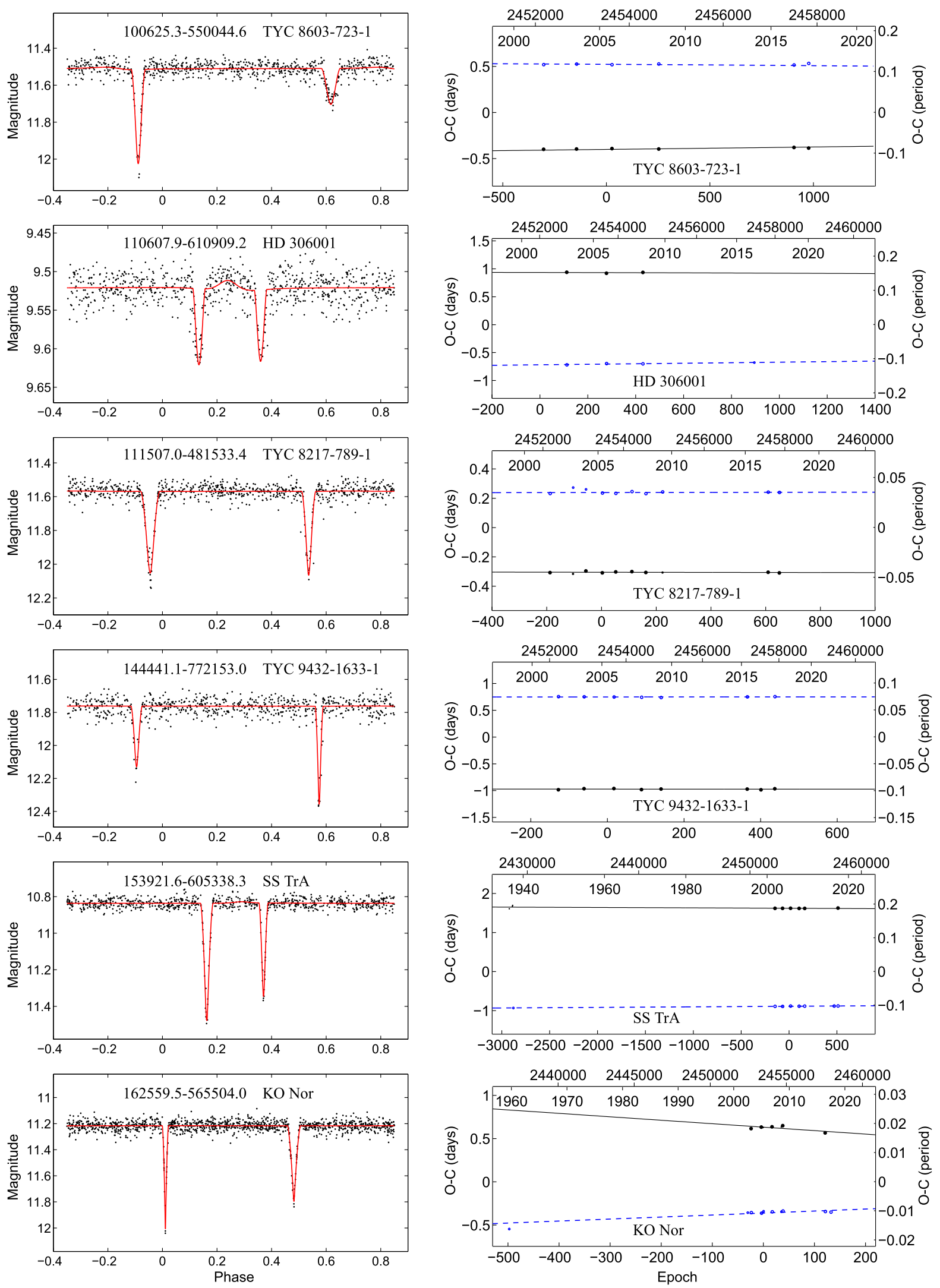

Fig. A.1. continued. 
P. Zasche et al.: The first study of 54 eccentric eclipsing binaries
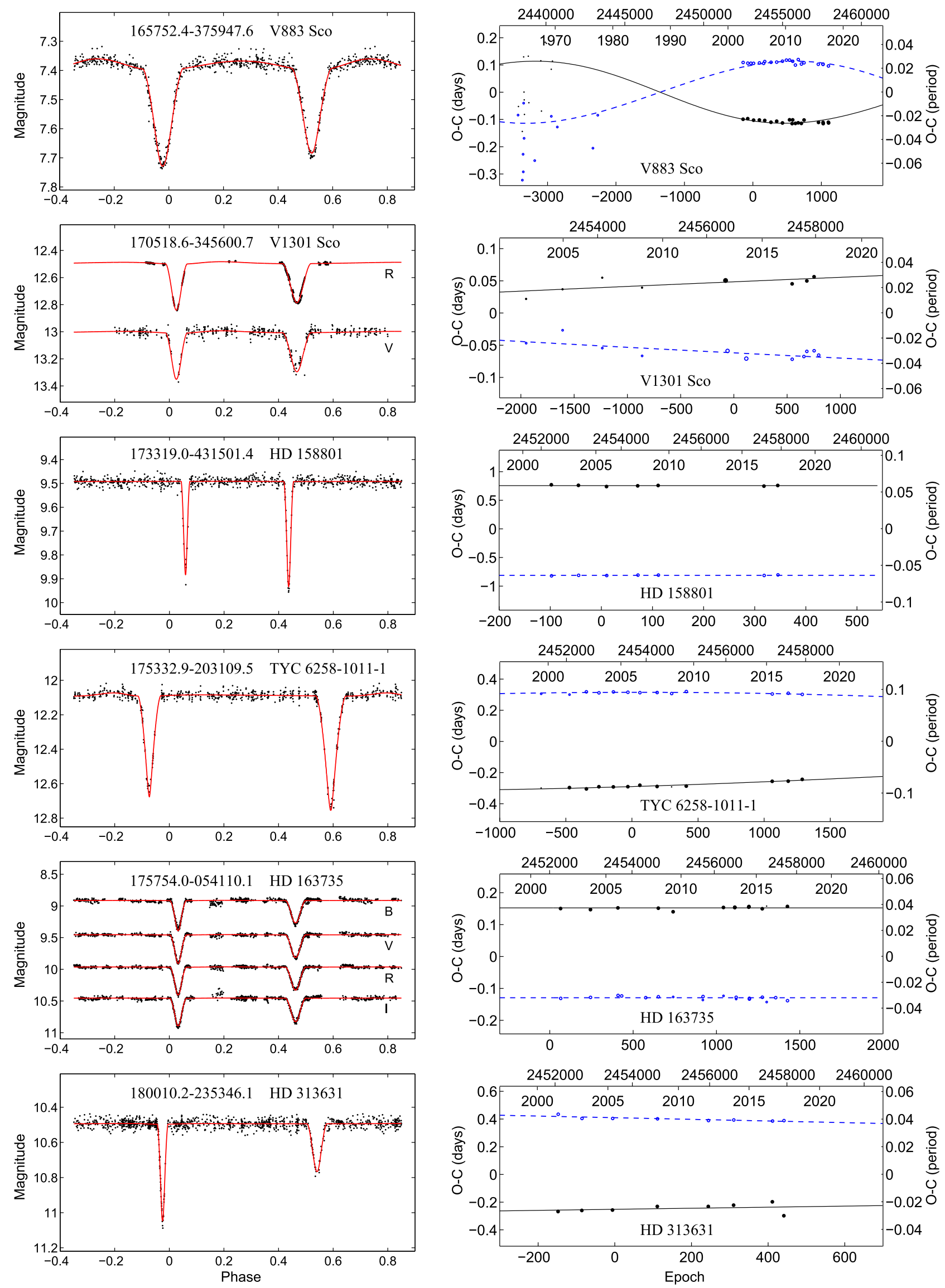

Fig. A.1. continued. 
A\&A 619, A85 (2018)
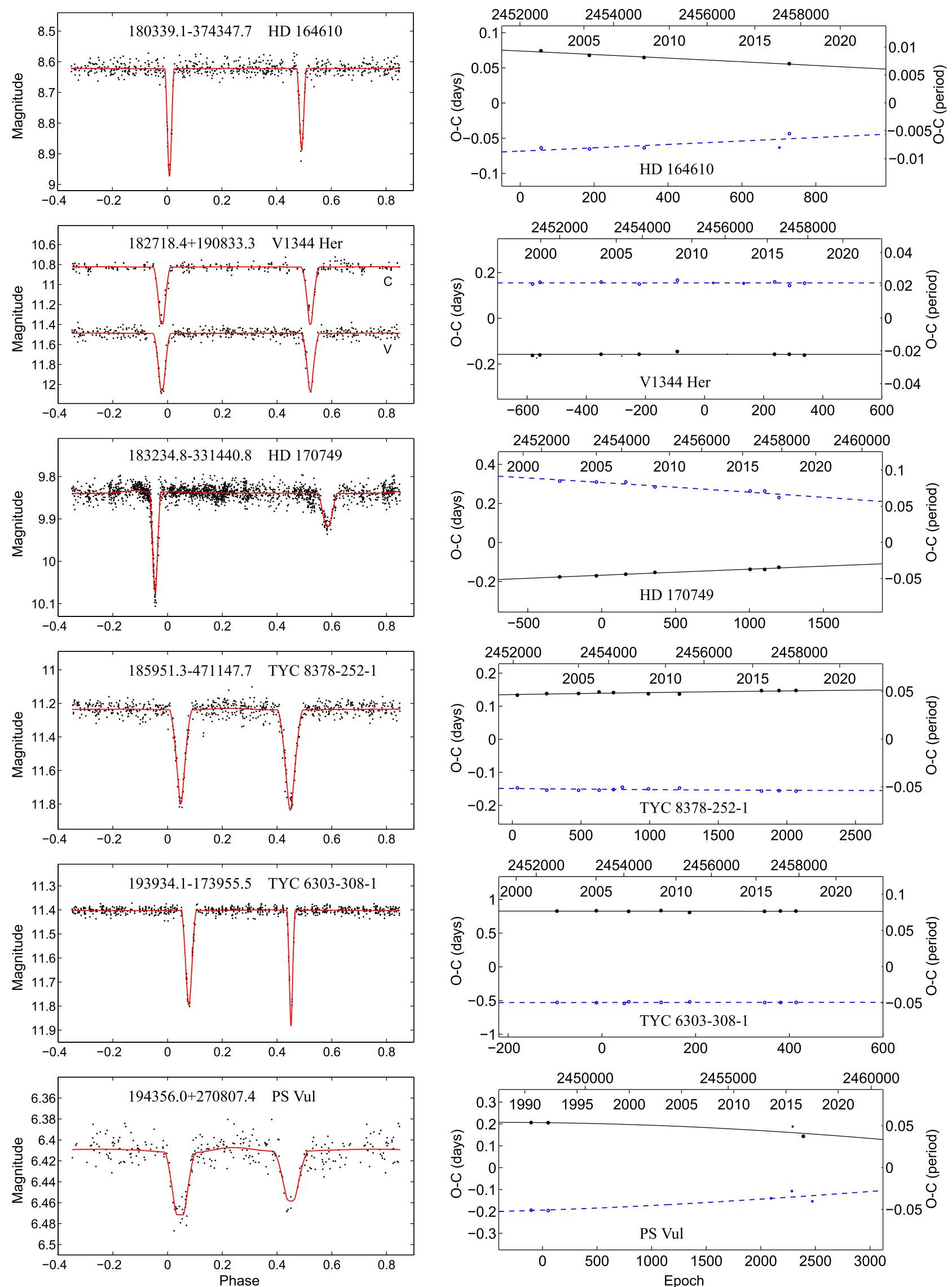

Fig. A.1. continued. 
P. Zasche et al.: The first study of 54 eccentric eclipsing binaries
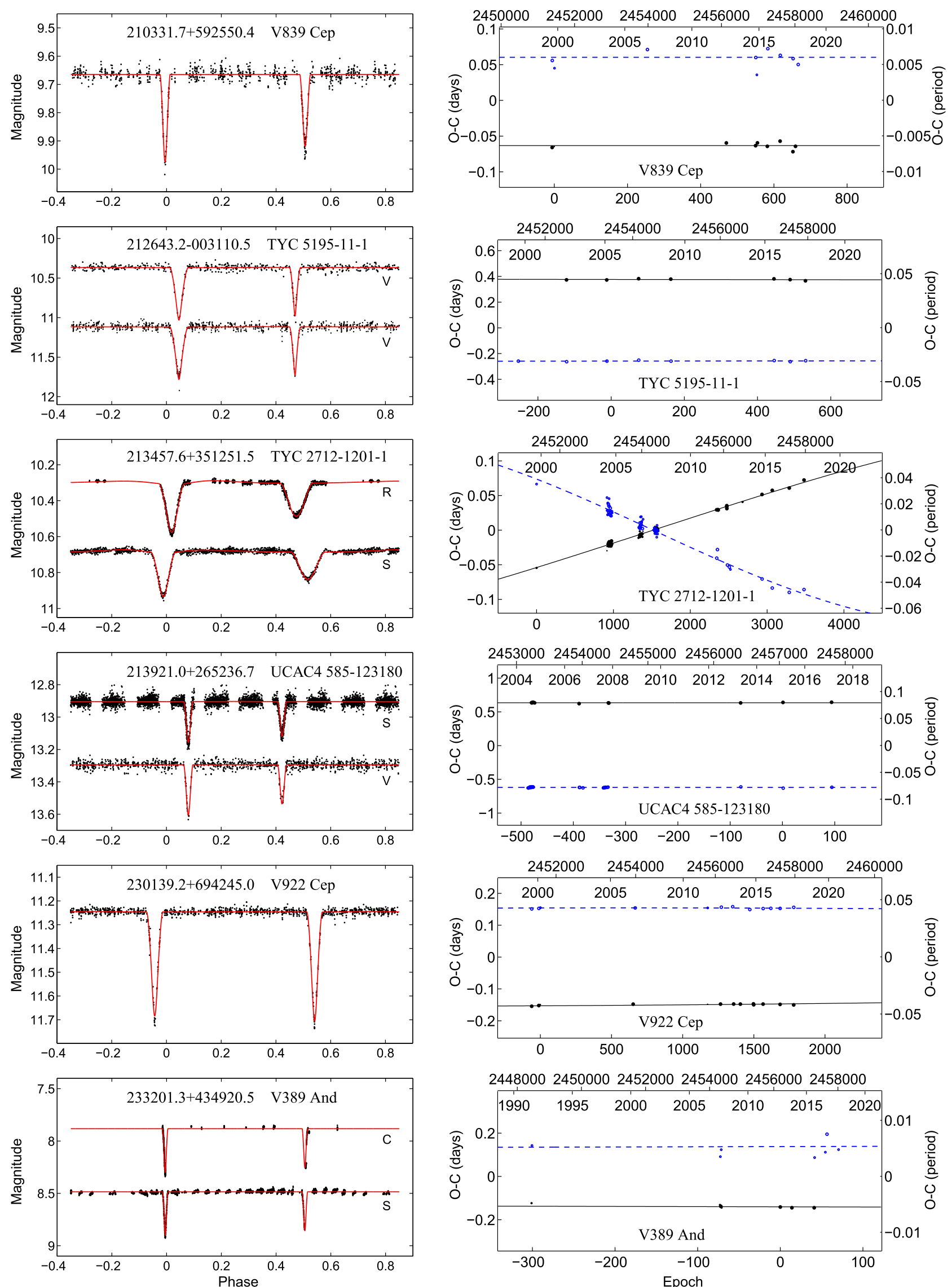

244800024500002452000245400024560002458000

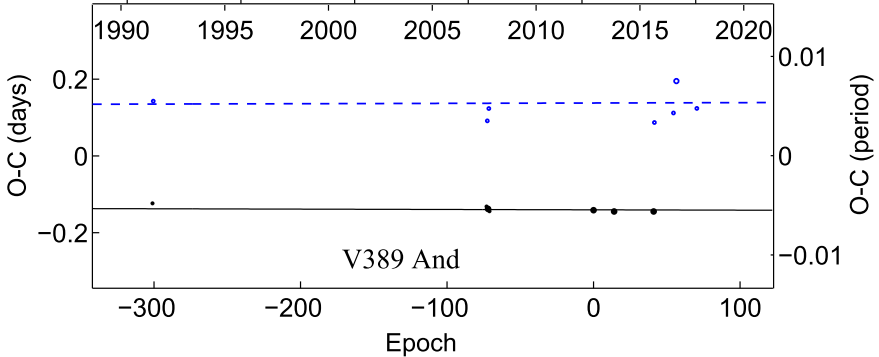

Fig. A.1. continued. 